\title{
Degradation Pathway Models for Photovoltaics Module Lifetime Performance
}

\author{
Nicholas R. Wheeler, Laura S. Bruckman, Junheng Ma, Ethan Wang, \\ Carl K. Wang, Ivan Chou, Jiayang Sun and Roger H. French

\begin{abstract}
L.S. Bruckman, N.R. Wheeler and R.H. French are with the Department of Material Science and Engineering, Case Western Reserve University, Cleveland OH, 44106 USA e-mail: (roger.french@case.edu).

J. Ma and J. Sun are with Department of Epidemiology and Biostatistics, Case Western Reserve University.

E. Wang and C.K. Wang are with Underwriters Laboratories. Ivan Chou is with Delsolar Co.
\end{abstract}

\begin{abstract}
Previously published accelerated testing data from Underwriter Labs, featuring measurements taken on 18 identical photovoltaic (PV) modules exposed to two stress conditions, were used to develop an analytical methodology. The results provide insight into active degradation mechanisms and pathways present in PV modules under accelerated testing conditions as indicated by statistically significant relationships between variables. Observed experimental results coincide with a domain knowledge based theoretical degradation pathway model informed by literature, and provide a basis for beginning to investigate the degradation modes and pathways truly present in modules and their effects on module performance over lifetime.

Index Terms-photovoltaics, statistical modeling, lifetime and degradation science, structural equation modeling.
\end{abstract}

\section{INTRODUCTION}

A recent U.S. Department of Energy workshop on Science for Energy Technologies [1] identified photovoltaics (PV) lifetime and degradation science (L\&DS) [2], [3], [4] as a critical scientific challenge for robust adoption of PV. The PVQA Task Force was developed as an international task force to work towards defining what is needed for lifetime qualification standards and tests [5]. Developing and defining useful lifetime qualification standards and tests is complicated because even single degradation modes, mechanisms and rates are not clearly understood and two-factor effects are even more complex. Therefore, a statistical and methodical approach is necessary to cross-correlate stressors, degrees of stress and degradation modes, mechanisms and rates for materials, components and systems. This cross-correlation can help provide a better understanding of degradation and lifetime performance in order to guarantee the minimal 25 year lifetime performance of PV modules [6].

\section{A. Lifetime and Degradation Science Approach}

The traditional method to determine the reliability of a system or component was to collect failure data and use a constant failure rate to account for product quality and environmental conditions. However, this approach is inaccurate at determining field failure rates and does not produce reliable lifetime predictions [7], [8]. In contrast, the physics of failure approach, based on failure modes and mechanisms as a function of the stress conditions, provides useful reliability information. The prognostics and health management approach assesses the reliability under application conditions and over lifetime. The combination of the physics of failure and prognostic approaches allows for continuous improvement of lifetime prediction [7], [8].

Lifetime approaches that consider system response, levels of response and degradation rates under stress conditions that include single, multi-factor, constant and cyclic conditions which vary by region are necessary. This lifetime approach must also cross-correlate real-world and accelerated lab based exposures [9], [10], [11]. An unbiased analytical approach that considers full system level modeling and a comprehensive degradation model, PV module lifetime and degradation science model (PVM L\&DS), that is refined iteratively by updated analysis is necessary in order to determine lifetime of current and new technologies.

A stress and response $(R(S))$ framework links stresses to observed responses, subsequent degradation and damage accumulation over the lifetime of PV materials, components and systems. Stressors can be characterized in terms of instantaneous stress level $(\sigma)$ and net or integrated stress $(S)$. A material's response $(R)$ to both $\sigma$ and $S$ can be described in Equation 1 as:

$$
R=f\left({ }^{\sigma} S\right)=\int f(\sigma) \sigma d t
$$

Degradation modes of materials can be elucidated by performing accelerated studies and a cross-correlation function can be used to predict the response observed in a system exposed to accelerated stressors compared to real-world conditions. The combination of single and multi-factor tests can lead to a better understanding of the synergistic effects of stress in a real-world environment. Response is therefore a function of the convolution of multiple stressors at their service-use conditions. Murray et al. exposed two grades of poly (methyl methacrylate) under two stress levels of full spectrum and ultraviolet light: a single-factor accelerated test. A constant "acceleration" factor was not observed for key degradation modes for the two stress conditions, demonstrating that the traditional search for a single acceleration factor is unreasonable. The $R(S)$ framework is a more versatile approach to encompass multiple stressors, stress levels and 
responses [9]. Mitigating key degradation modes and lifetime penalties can increase a PV module's lifetime performance.

\section{B. Statistical and Domain Analytics: L\&DS Analytics}

A PVM L\&DS model can provide knowledge of rankordered degradation modes for different climatic zones and stress conditions, which can highlight modes contributing to performance and lifetime for a particular use condition. A statistically valid approach is necessary for the development of a reliable PVM L\&DS model for accurate lifetime prediction. The statistical analysis requires a large data set in terms of variables and sample sizes, both for groups of measurements at single time points as well as the total number of measured time points over the entire timeframe of the study. Statistically significant relationships (SSRs) need to be elucidated between different stressors and degradation modes and pathways. Statistics such as R-Squared Values and P-Values can be used to assess the strength of these relationships between measured response variables. Errors such as random, measurement, systematic and method errors should be incorporated into the methodology to remove bias from the resulting model [12].

After statistical analysis is performed, domain knowledge is used to understand the correlations indicated and other results of the statistical analysis. Observations can be framed in terms of known mechanisms and pathways as well as a particular system response. Domain knowledge can be used to identify the probable active and quiescent pathways. Many different pathways can be activated by different stressors or stress levels and need to be incorporated into the PVM L\&DS model. Figure 1 shows a theoretical PV module degradation pathway model, which is based on observations from both real-world exposed modules and modules subjected to accelerated test conditions [13], [14], [15], [16], [17], [18]. When module failure modes can be attributed to processes that are only activated under accelerated exposure conditions or accelerated exposure conditions are chosen that fail to activate pathways observed in field-exposed modules, it signals inappropriate exposure conditions with little basis in reality. To assess which testing conditions recreate sensible real-world module degradation modes for the prediction of module performance, the deciding factor will be which degradation pathways are activated by the chosen stress levels using the stress and response framework and lifetime and degradation science [9].

The iterative nature of the PVM L\&DS model allows for the continuous refinement of the model. As more knowledge is gained through accelerated exposure studies and real-world data and as new technologies are incorporated into the model, the correlations and predictions will become stronger over time for a wider range of module designs; therefore producing a predictive, statistically significant prognostic model. Proper documentation of the limitations and scope of a predictive model is important to ensure that a model is providing useful information on lifetime [19].

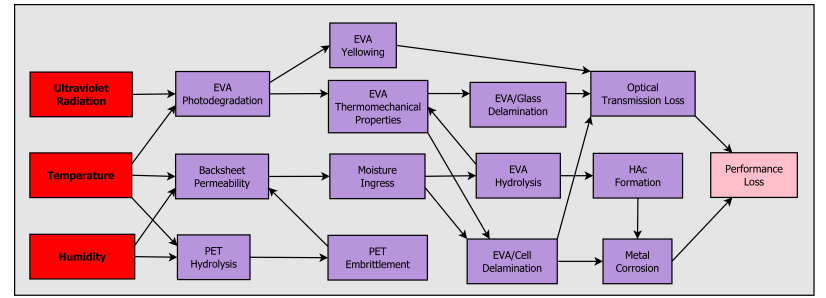

Fig. 1. Example theoretical PV module performance degradation pathways

\section{UL PREVIOUS RESUlts}

The data used for the statistical modeling was published by E. Wang et. al. [20]. Eighteen commercially available polycrystalline 60-cell solar PV modules made with FPE (fluoropolymer, polyester, and EVA) backsheets were fabricated under identical processing conditions by DelSolar [21]. Eight PV modules were subjected to damp heat (DH) aging, eight modules were exposed to UV and two modules were used as control samples and not exposed. Test laminates of ethylenevinyl acetate (EVA) and glass were fabricated based on the module design and lamination process and were used for interfacial adhesion tests. The edge of these test laminates were sealed with a waterproof and temperature resistance acrylic foam tape to avoid excess moisture ingress.

There were no specified variations in the PV modules used for the experiments and the intention of the work was not to analyze differences in results between constrasting PV modules. By keeping the choice of PV module a constant and varying the exposure conditions rather than comparing performances between multiple module types, a wider range of degradation modes can be observed and characterized within a single PV module system. The scope of the data collected for each variable under each exposure type over time can be seen in Figure 2

\section{A. Damp Heat Exposure}

Damp heat exposure entailed $85^{\circ} \mathrm{C}$ temperature and $85 \%$ relative humidity and is described in test 10.13 of IEC 61215 Ed.2. [22]. The FPE PV modules and test laminates were aged in damp heat conditions four times longer than the IEC standard of 1000 hours. Two of the original eight modules were removed from the chamber at each 1000-hour time increment and destructively disassembled, and packaging materials were collected and tested, until a maximum exposure time of 4000 hours [20].

\section{B. UV Irradiance Exposure}

The UV exposure was similar to test 10.10 of IEC 61215 Ed.2, [22] but with higher light intensity, approximately 80 $\mathrm{W} / \mathrm{m}^{2} \mathrm{UV}$ irradiance at $280-400 \mathrm{~nm}$ wavelengths plus an additional $15 \%$ of the total irradiance at the backside of the PVMs and test laminates. The module temperature was controlled at $60^{\circ} \mathrm{C}$, and the relative humidity was uncontrolled. The total irradiance, until 4000 hours, was about 20 times higher than IEC standard of $15 \mathrm{kWh} / \mathrm{m}^{2}$. Two of the original eight 
modules were removed from the chamber at each 1000-hour time increment and destructively disassembled, and packaging materials were collected and tested, until a maximum exposure time of 3000 hours [20].

\section{PV Module Responses}

Experiments were performed on the module, component and mechanistic level for modules from each of the exposure conditions in order to extract information on each of these levels. This provides a more in-depth study of the degradation modes and how these modes affect components and the overall module performance. In addition, performing the same experiments for both damp heat and UV exposures leads to an understanding of which degradation modes and pathways are active during each type of stress. The results of these experiments are directly related to time of exposure since conditions were held constant in the damp heat and UV exposures.

\section{System Responses}

Three system responses were measured for each of the 16 PV modules which included peak power (Pmax), fill factor $(F F)$ and wet insulation resistance (WetIns). The systemlevel responses were measured every 500 hours for both the damp heat and UV experiments from 0 to 2500 hours, and then every 250 hours from 2500 to 4000 hours for damp heat and 2500 to 4250 hours for UV experiments [20]. P $\max$ is the peak power of a photovoltaic module, and is determined by measuring current and voltage while varying resistance under defined illumination. The power reduction is driven by reduced $F F$ (a metric describing the ideality of the shape of the current and voltage curve) and increased series resistance. These reductions are initially interpreted as corrosion taking place at the electrical interconnects [23], [24], [25]. Wet insulation resistance testing according to IEC 61215 [22] is intended to verify that a PV module's packaging materials have sufficient electrical insulation properties to reduce the possibility of fire and electric hazards, even when the module is wet.

\section{E. Unit Experiments}

Ten component-level unit experiments were performed on the modules and one unit experiment was performed on the test laminates. These experiments can be separated into two categories: performance (peel strength of the EVA-glass interface in the test laminates (Peel), EVA modulus ( $M o d)$, EVA glass transition temperature $\left(T_{g}\right)$, water vapor transmission rate of the backsheet $(W T V R)$ and differential scanning calorimetry of EVA $(D S C)$ ) and mechanistic (two IR measurements of EVA hydrolysis $I R E V A$ and $I R 2$, thermogravimetric analysis of EVA (TGA), EVA acetic acid content (Hac), and 2 IR measurements of backsheet PET hydrolysis IRBS1 and $I R B S 2)$. The unit experiments were performed on component materials and subsystems of dissassembled modules every 1000 hours of testing [20].

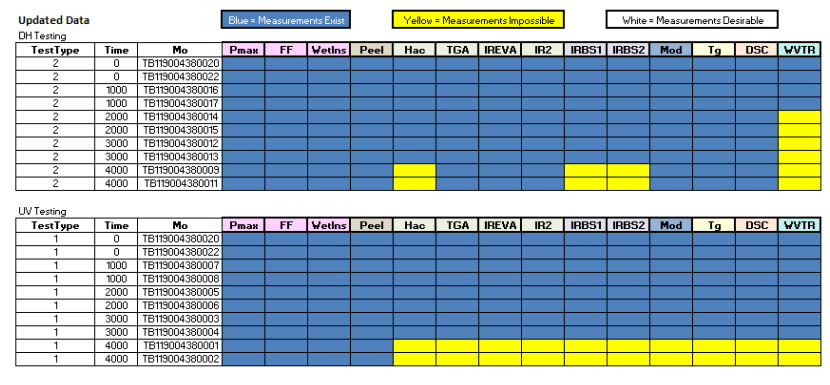

Fig. 2. Summary of data from PV modules for each of the system and unit data for the DH (top) and the UV (bottom) experiments. The blue squares indicates data that was taken for the time point and the yellow squares indicate data that was not collected [20].

\section{L\&DS AnAlytics: Methods}

\section{A. Data Determination}

Two unexposed modules were disassembled and unit-level variable measurements taken to capture a shared baseline between the DH and UV exposures. Two additional modules per stress condition were disassembled at each 1000-hour interval (up to 4000 hours for the DH exposure and 3000 hours for the UV exposure) for measurements of the unitlevel variables. Although the system-level variables (P $\max$, $F F$, WetIns) were measured more frequently than 1000-hour intervals, they share only these time points with the unit-level variable measurements. Only these shared time points allow comparison between measurements of different variables from identical modules, which is the basis of the coincidence of observations necessary for this statistical analysis.

The number of measurements at each time point for each variable was not consistent between different variables. Some of the variables were measured multiple times (2-6 per harvested module at each time point), while others were only measured once. Additionally, individual measurements at identical time points between different variables are only comparable to one another on the basis of their sample origin (which module they came from); they do not correspond directly to one another even if the number of measurements is identical. Therefore, in the event of multiple measurements of a single variable from a single module at a single time point, the measurements were averaged to a single value to enable comparison between variables.

Variables differ slightly in regards to how the results of the measurements were interpreted into single discrete values. The Pmax, FF, WetIns, WVTR, Hac, Peel and $T_{g}$ measurements each provide a single value which can be used without modification. The test results of IREVA, $I R 2, I R B S 1$ and $I R B S 2$ are absorption spectra, which are interpreted into specific meaningful ratios of integrated absorption bands to provide singular values. The $T G A$ test provides a curve of sample weight versus temperature, portions of which (characterized by changes in the slope of the curve) represent losses of specific chemical groups. The total weight lost over a particular interval corresponds to the amount of acetate side groups present in the polymer, which functions 
as a single discrete value for each $T G A$ curve. The $D S C$ test provides a curve describing the change of heat flow into a sample over a scanned temperature range. Changes in the heat flow correspond to changes in the degree of crystallinity present in the sample. The degree of crystallinity can be quantified, which functions as a single discrete value for each $D S C$ curve. Dynamic mechanical analysis $(M o d)$ results in a curve describing changes in material modulus over a scanned temperature range. A single temperature was chosen $\left(71.363^{\circ} \mathrm{C}\right)$ and the modulus at that point used to describe each dynamic mechanical analysis curve as a single discrete value.

\section{B. Variable Selections}

In a typical data analysis problem, there are predictors and response variables. Often, some predictors have little or no influence on the response variable. The procedure to identify those predictors or to select the "best" subset of the predictors, such that the statistical model based on this subset of predictors is as significant as the full model based on all the predictors, is called variable selection. This data set features small $n$ and large $\mathrm{p}$ data (meaning a small number of observations for a comparatively large number of variables) although this is not the typical case. The number of variables that can be considered in the statistical analysis is restricted by the number of observations per variable according to the formula $n-2=v$, where $n$ represents the number of observations and $v$ represents the number of variables that can be included in the analysis. In this case $n=8$ observations, so only 6 variables can be included. This means more variables exist than can be simultaneously included into the modeling, and so variables must be chosen on the basis of measurement confidence and domain knowledge based expectation of significance to the overall model. Due to the limited amount of data, domain knowledge has been used to select contrasting subsets of predictors to use for the initial variable selection step of constructing the statistical pathway models.

\section{Mapping to Domain Experience}

The models relating the variables should be checked for consistency with domain knowledge about the phenomena being measured. With respect to time, chemical and physical processes are typically either linear or exponential, so if a polynomial model is found to provide the best fit, this could indicate that there are change points resulting from the influence of combined effects. Additionally, phenomena anticipated to be exponential could appear linear if test conditions are not aggressive enough to elicit sufficient response over the total measurement time. Domain knowledge should always be applied to the results of the statistical analysis; relationships indicated statistically that violate domain knowledge based expectations, or domain knowledge based expectations that are not reflected by the statistical analysis, both indicate that further investigation is required.

\section{Statistical Modeling}

Presently two principles have been used for investigating relationships between the chosen initial subsets of variables.
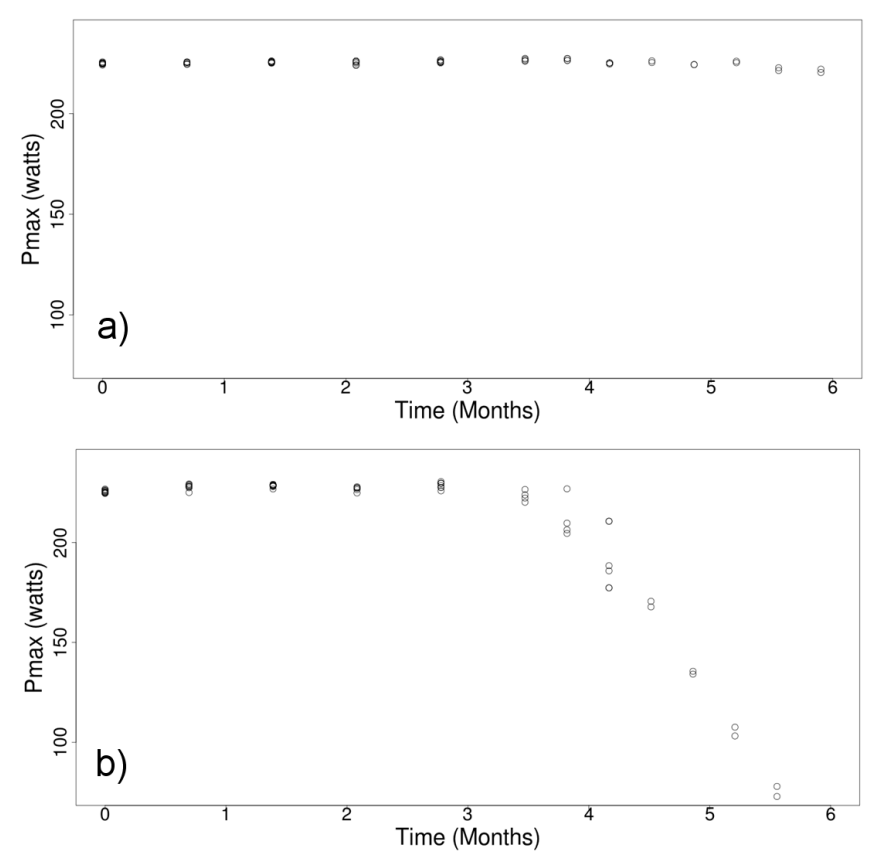

Fig. 3. The system level response P $\max$ showed minimal response to a) UV testing conditions and a drastic response to b) damp heat testing conditions.

The first principle examines only univariate relationships based on a selection of known functional forms directly from one variable to another, and performs iterative variable selection steps on the basis of a 0.2 cutoff of adjusted-r-squared value. The second principle examines the collective additive influence of variables upon one another utilizing Akaike Information Criterion (AIC) values to perform stepwise variable selections iteratively to indicate networks of relationships between the variables [26].

The development of the PVM L\&DS model allows for the refinement of the model with more data over time; therefore, with each iteration of the modeling procedures in light of additional data, increasingly accurate statistical results will be obtained.

\section{L\&DS ANALYTICS: RESUltS}

\section{A. System Level Responses}

For both damp heat and UV, the system-level responses examined were P $\max$ and $F F$. WetIns was not examined due to an insufficient number of measurements to compensate for evident outliers in the data. The UV exposures elicited minimal response in either of the system level variabless. For damp heat exposures both system level variabless showed dramatic responses, though P $\max$ was found to contain more information than $F F$ on the basis of quantity and statistical quality (as determined by R-Squared and P-Values) of relationships to other variables.

\section{B. Selected Variables}

Mechanistic unit-level predictors were chosen for the initial subset of predictors because they could be attributed to 
TABLE I

RESPONSE VARIABLES INCLUDED IN THE MODELING.

\begin{tabular}{|c|c|}
\hline $\begin{array}{c}\text { System Level } \\
\text { Response }\end{array}$ & Unit Level Variables \\
\hline Pmax or FF & Hac or TGA,IREVA or IR2, IRBS1 or \\
IRBS 2
\end{tabular}
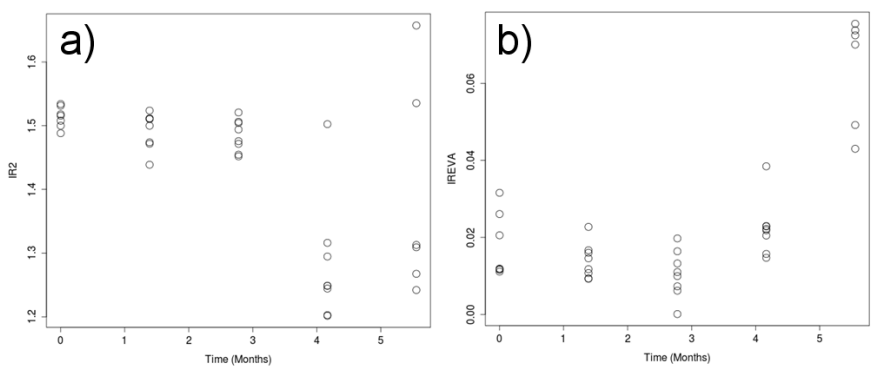

Fig. 4. As a measure of EVA hydrolysis, a) $I R 2$ was found to be preferable to b) IREVA due to its information content (the quantity and quality of statistical relationships to other variables, based upon R-Squared and PValues).

specific, known phenomena. Based upon domain knowledge and measurement confidence, in addition to time the variables examined in the modeling are listed above. (Table [ ] )

'Redundant' unit level predictors (those aimed at measuring identical mechanistic phenomena) were also compared to one another on the basis of their information content (the quantity and quality of statistical relationships to other variables, based upon R-Squared and P-Values).

As a specific example, IR2 and $I R E V A$ both tracked EVA hydrolysis in the encapsulant, examining changes in the presence of two chemical functional groups related to this phenomena. Though both of these variables showed significant responses (Figure 4), IR2 was found to be preferable to $I R E V A$ on the basis of its information content when these variables were interchanged in the modeling process. Hac and $I R B S 1$ were similarly found to be preferable to their counterparts for the purposes of statistical modeling.

\section{Statistical Models}

Of the 32 possible combinations of the chosen variables that were modeled with each of the two previously described principles of variable selection, the Principle 1 modeling technique applied to the variables Time, Hac, IR2, IRBS 1 , and Pmax was found to possess the most information content and mapped well to domain knowledge [26]. A full prognostic model was not developed due to some limitations in the data set, though valuable observations about PV module response to damp heat testing conditions were evident from the statistical analysis.

\section{L\&DS ANALYTICS: DISCUSSIONS}

\section{A. UV Testing Responses}

UV testing conditions created minimal response in the system level performance variables over the $3000 \mathrm{hr}$ exposure period. This made further analysis of the modules exposed

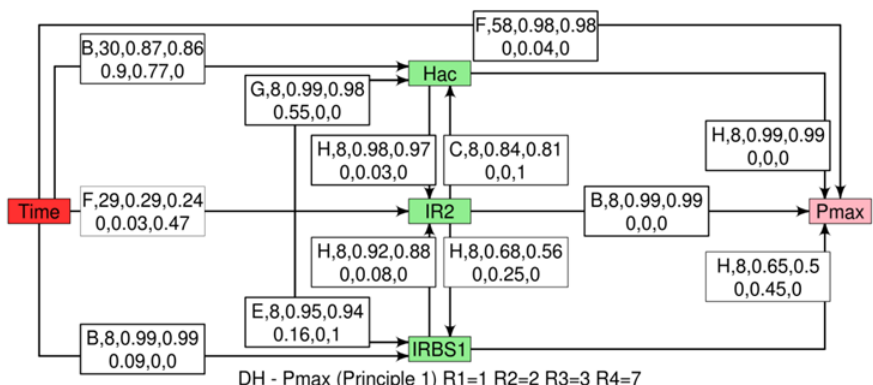

Fig. 5. The statistical pathway model generated using Principle 1 for the variables Time, Hac, IR2, IRBS1, and Pmax was found to contain the most information content and mapped well to domain knowledge[26].

to UV testing impossible because there were not adequate responses among the variables for the purposes of statistical correlation. Apparent relationships between the variables were statistically weak and attributable to random fluctuation, there was inadequate signal above the noise for the purposes of sound statistical analysis.

\section{B. Damp Heat Testing Responses}

Damp heat testing conditions created dramatic response in the system level performance variables over the $3000 \mathrm{hr}$ exposure period, with an evident change point occuring after an initial period of linearity. This is interpreted as a period of damage accumulation prior to a runaway degradation process. Many statistically significant relationships were present between variables that fit known functional forms of mechanistic degradation processes, which allowed for meaningful interpretation of the results.

\section{Information Content of Variables}

Unit and system level variables from the damp heat exposures showed responses consistent with domain knowledge expectations. Multiple variables measuring a single mechanistic response are redudant for the statistical pathway model analysis, as multiple variables measuring the exact same response should not be included together in the modeling process. In these cases, one of the variables is chosen on the basis of its information content, with a preference for the overall combination of variables that displays the highest number of statistically significant relationships between all the variables in the model. Redundancy in variables is highly desireable for the purposes of maximizing the information content of the final model in this manner.

\section{Statistical Pathway Model Observations}

The statistical pathway model with the highest information content for the damp heat results featured the variables Time, $H a c, I R 2$, IRBS1, and P max. Observations can be made about the degradation of modules exposed to damp heat conditions by examining the statistical relationships between these variables. 


\section{E. Indicated Pathway of Degradation}

Relationships between system and unit level responses to damp heat indicate sequential degradation steps leading to overall system performance loss. This is interpreted as temperature and humidity induced PET hydrolysis in the backsheet enhancing moisture ingress, facilitating EVA hydrolysis and acetic acid generation, leading to the corrosion of cell metallization and a loss of Pmax. This is consistent with known mechanisms and the theoretical degradation pathway model.

These effects could be caused by damp heat, but it is unclear if this represents reality for a deployed module. Damp heat testing is intended as an accelerated means to assess a module design's ability to withstand the stresses of its use environment. It is possible, however, that some mechanisms activated by an overly 'accelerated' test are not present during real-world operation, leading to misinformed module design, faulty prognostics and ultimately wasted resources.

\section{CONCLUSION}

Traditional lifetime reliability methods do not provide a statistical means to accurately predict lifetime of PV modules. To gain insights into lifetime through accelerated testing, the information collected needs to facilitate sound statistical practice. Accelerated testing protocols should be constructed to create sufficient degradation for analysis, while still activating modes expected in the real world. Multiple tests investigating the same degradation mode reveal the combination of responses providing the most information content. SSRs among variables indicate active degradation mechanisms and pathways present within the system as a response to particular stress conditions. Degradation modes activated by overly harsh conditions need to be studied further to identify and characterize true degradation modes relevant to use conditions.

\section{ACKNOWLEDGMENTS}

The authors acknowledge funding from Underwriter Laboratories. Research was performed at the SDLE Center at Case Western Reserve University, funded through the Ohio Third Frontier, Wright Project Program Award Tech 12-004.

\section{REFERENCES}

[1] J. Hemminger, "From quanta to the continuum: Opportunities for Mesoscale Science," A Report from the Basic Energy Sciences Advisory Committee, Tech. Rep., 2012.

[2] M. P. Murray, D. Gordon, S. A. Brown, W.-C. Lin, K. A. Shell, M. A. Schuetz, S. Fowler, J. Elman, and R. H. French, "Solar radiation durability framework applied to acrylic solar mirrors," Proc. SPIE 8112, Reliability of Photovoltaic Cells, Modules, Components, and Systems IV, 811203 (September 13, 2011), pp. 811203-811203-10, 2011. [Online]. Available: +http://dx.doi.org/10.1117/12.893827

[3] R. French, M. Murray, W.-C. Lin, K. Shell, S. Brown, M. Schuetz, and R. Davis, "Solar radiation durability of materials components and systems for low concentration photovoltaic systems," in Energytech, 2011 IEEE, May 2011, pp. $1-5$.

[4] R. French, J. Rodríguez-Parada, M. Yang, M. Lemon, E. Romano, and P. Boydell, "Materials for concentrator photovoltaic systems: Optical properties and solar radiation durability," in AIP Conference Proceedings, vol. 1277, 2010, p. 127.

[5] "International PV Module Quality Assurance Task Force, established by NREL, AIST, PVTEC, U. S. DOE, EU JRC, SEMI PV Group to develop PV Lifetime Qualification Testing," 2012.
[6] J. Hemminger, G. Crabtree, and A. Malozemoff, "Science for energy technology: Strengthening the link between basic research and industry," A report from the Basic Energy Sciences Advisory Committee, US Department of Energy, 2010.

[7] M. G. Pecht, Introduction. John Wiley \& Sons, Inc., 2008, pp. 1-24. [Online]. Available: http://dx.doi.org/10.1002/9780470385845.ch1

[8] —_, Physics-of-Failure Approach to PHM. John Wiley \& Sons, Inc., 2008, pp. 73-84. [Online]. Available: http://dx.doi.org/10.1002/ 9780470385845.ch4

[9] M. P. Murray, L. S. Bruckman, and R. H. French, "Photodegradation in a stress and response framework: Poly(methyl methacrylate) for solar mirrors and lens," Journal of Photonics for Energy, vol. 2, no. 1, pp. 022 004-022 004, 2012. [Online]. Available: +http://dx.doi.org/10.1117/1.JPE.2.022004

[10] M. Murray, L. Bruckman, and R. French, "Durability of acrylic: Stress and response characterization of materials for photovoltaics," in Energytech, 2012 IEEE, May 2012, pp. 1 -6.

[11] L. Bruckman, M. Murray, S. Richardson, S. Brown, M. Schuetz, and R. French, "Degradation of back surface acrylic mirrors: Implications for low concentration and mirror augmented photovoltaics," in Energytech, 2012 IEEE, May 2012, pp. 1-4.

[12] R. Bagozzi and Y. Yi, "Specification, evaluation, and interpretation of structural equation models," Journal of the Academy of Marketing Science, vol. 40, no. 1, pp. 8-34, 2012. [Online]. Available: http://dx.doi.org/10.1007/s11747-011-0278-x

[13] F. Pern, "Factors that affect the EVA encapsulant discoloration rate upon accelerated exposure," in Photovoltaic Energy Conversion, 1994., Conference Record of the Twenty Fourth. IEEE Photovoltaic Specialists Conference - 1994, 1994 IEEE First World Conference on, vol. 1, Dec 1994, pp. $897-900$ vol.1.

[14] F. Pern and S. Glick, "Photothermal stability of encapsulated Si solar cells and encapsulation materials upon accelerated exposures," Solar Energy Materials and Solar Cells, vol. 61, no. 2, pp. 153-188, 2000.

[15] D. King, W. Boyson, and J. Kratochvill, "Photovoltaic array performance model. Sandia National Laboratories, Albuquerque, New Mexico, 87185-0752," 2004.

[16] D. L. King, M. A. Quintana, J. A. Kratochvil, D. E. Ellibee, and B. R. Hansen, "Photovoltaic module performance and durability following long-term field exposure," Progress in Photovoltaics: Research and Applications, vol. 8, no. 2, pp. 241-256, 2000. [Online]. Available: http://dx.doi.org/10.1002/(SICI) 1099-159X(200003/04)8:2〈241::AID-PIP290 $>3.0 . C O ; 2-D$

[17] W. Gambogi, "Comparative performance of backsheets for photovoltaic modules," in 25th European Photovoltaic Solar Energy Conference and Exhibition (EU PVSEC), 2010.

[18] J. Del Cueto and T. McMahon, "Analysis of leakage currents in photovoltaic modules under high-voltage bias in the field," Progress in Photovoltaics: Research and Applications, vol. 10, no. 1, pp. 15-28, 2002.

[19] J. Elerath and M. Pecht, "IEEE 1413: A standard for reliability predictions," Reliability, IEEE Transactions on, vol. 61, no. 1, pp. $125-129$, March 2012.

[20] E. Wang, H. E. Yang, J. Yen, S. Chi, and C. Wang, "Failure modes of evaluation of pv module via materials degradation approach," in $P V$ Asia Pacific Expor and Conference, 2012. Elsevier Science Direct, in press.

[21] DelSolar Co., Ltd. Headquarters 6 Kebei $2^{\text {nd }}$ Road, Shunan Science Park, Zhunan Township, Miaoli Country 35053, Taiwan, R. O. C.

[22] IEC 61215, "IEC Standard 61215 second edition-Crystalline silicon terrestrial photovoltaic (PV) modules-design qualification and type approval," ASTM International, 2005, IEC 61215:2005(E).

[23] B. Ketola and A. Norris, "Degradation mechanism investigation of extended damp heat aged PV module," in 26th EUPVSEC, 2011.

[24] K. Whitfield, A. Salomon, S. Yang, and I. Suez, "Damp heat versus field reliability for crystalline silicon," in Photovoltaic Specialists Conference (PVSC), 2012 38th IEEE, June 2012, pp. 001864 -001870.

[25] S. Sakamoto, T. Kobayashi, and S. Nonomura, "Epidemiological analysis of degradation in silicon photovoltaic modules," Japanese Journal of Applied Physics, vol. 51, p. 10NF03, 2012. [Online]. Available: http://jjap.jsap.jp/link?JJAP/51/10NF03/

[26] L. S. Bruckman, N. R. Wheeler, J. Ma, E. Wang, C. K. Wang, I. Chou, J. Sun, and R. H. French, "Statistical and domain analytics applied to PV module lifetime and degradation science," IEEE Open Access, Invited Paper, in press. 